\title{
Toma de decisiones en el sector turismo mediante el uso de Sistemas de Información Geográfica e inteligencia de negocios
}

\author{
Geographic information systems and business intelligence in decision making in the \\ tourism
}

\section{Tomada de decisão no setor de turismo através do uso de Sistemas de Informação Geográfica e inteligência de negócios}

\author{
Carlos-Fernando Barrera-Narváez ${ }^{1}$ \\ Juan-Sebastián González-Sanabria ${ }^{2}$ \\ Gustavo Cáceres-Castellanos ${ }^{3}$ (D)
}

Recibido: enero 2020

Aceptado: abril 2020

Para citar este artículo: Barrera-Narváez, C.F., González-Sanabria, J.S., Cáceres-Castellanos, G. (2020). Sistemas de información geográfica e inteligencia de negocios en la toma decisiones en el sector turismo. Revista Científica, 38(2), 160-173. https://doi.org/10.14483/23448350.15997

\section{Resumen}

Actualmente, la inteligencia de negocios está presente en cualquier proceso de análisis de datos, principalmente, en casos en los que se evalúa la viabilidad de mercados, la inclusión de nuevos productos o la actividad que permite conocer los hábitos de consumo de las personas, lo cual muestra ventajas evidentes. El sector turístico tiene como fin proyectar y potencializar a las regiones, según las necesidades o intereses de las personas, por lo que los modelos de inteligencia de negocios son de fundamental utilidad para cumplir con dicho objetivo. Adicionalmente, al integrar a la inteligencia de negocios, aspectos relacionados con el espacio para determinar la cercanía o el desplazamiento de las personas entre regiones, mediante el uso de Sistemas de Información Geográfica (SIG), se puede obtener una representación visual del estado del turismo en una región de interés e incluso evaluar la integración de regiones cercanas y ofrecer planes en conjunto para tener beneficios y progreso conjunto. Este artículo presenta una revisión sistemática de literatura sobre el uso de sistemas de información geográfica y de inteligencia de negocios para la toma de decisiones apoyadas en el sector turismo; para lo cual se analizaron artículos publicados durante 2015-2019 en las bases de datos Scopus e IEEE. Se obtuvo como resultado que durante dicho periodo la tendencia en investigaciones relacionadas con la temática de uso de los GIS en turismo ha sido creciente. Se concluye que gracias a los avances tecnológicos con el uso de las WebGIS y Open Source GIS, los gerentes de empresas turísticas han podido construir sistemas de Información de Recursos Turísticos.

1. Universidad Pedagógica y Tecnológica de Colombia. Tunja-Boyacá, Colombia. carlos.barreranarvaez@uptc.edu.co

2. Universidad Pedagógica y Tecnológica de Colombia. Tunja-Boyacá, Colombia. juansebastian.gonzalez@uptc.edu.co

3. Universidad Pedagógica y Tecnológica de Colombia. Tunja-Boyacá, Colombia. gustavo.caceres@uptc.edu.co 
Palabras clave: inteligencia de negocios, sistemas de soporte a la toma de decisiones, turismo, sistemas de información geográfica y SIG.

\section{Abstract}

Currently, business intelligence is present in any process of data analysis, mainly due to the advantages that the moment of evaluation of market viability, inclusion of new products, and if any activity that requires knowing people's consumption habits has Within these activities, there is tourism, since, for this sector it is also important to be able to make decisions and focus on potentializing the regions according to the needs or interests of the people. In addition, by integrating business intelligence, aspects related to space, to determine proximity, or the displacement of people between regions, through the use of Geographic Information Systems (GIS) you can obtain a visual representation of the state of tourism in a region of interest and even evaluate the integration of the affected regions and offer planes together to have benefits and joint progress. In this sense, this article presents a systematic review of literature on the use of geographic information systems and business intelligence for decision-making supported in the tourism sector; for which articles published during the period 2015 to 2019 in the Scopus and IEEE databases will be analyzed. It was obtained as a result that during the last years (2015-2019) the trend in research related to the use of GIS in tourism has been increasing. It is concluded that thanks to technological advances, the use of, WebGIS, Open Source GIS, tourism business managers have built Tourist Resources Information systems. Keywords: Business Intelligence, Decision Support System, Tourism, Geographic Information System, GIS.

\section{Resumo}

Atualmente, a inteligência comercial está presente em qualquer processo de análise de dados, principalmente nos casos em que é avaliada a viabilidade de mercados, a inclusão de novos produtos ou a atividade que permite que as pessoas conheçam os hábitos de consumo das pessoas, o que mostra vantagens óbvias. O setor de turismo tem como objetivo projetar e capacitar as regiões, de acordo com as necessidades ou interesses das pessoas, para que os modelos de inteligência de negócios sejam de utilidade fundamental para atingir esse objetivo. Além disso, ao integrar a inteligência de negócios, aspectos relacionados ao espaço para determinar a proximidade ou o deslocamento de pessoas entre regiões, através do uso de Sistemas de Informação Geográfica (SIG), podem ser obtidos uma representação visual do estado do turismo. em uma região de interesse e até avaliar a integração de regiões próximas e oferecer planos conjuntos para obter benefícios e progresso conjunto. Este artigo apresenta uma revisão sistemática da literatura sobre o uso de sistemas de informações geográficas e inteligência de negócios para tomada de decisão apoiada no setor de turismo; para os quais foram publicados artigos publicados durante 2015-2019 nas bases de dados Scopus e IEEE. Foi obtido como resultado que, durante esse período, a tendência nas pesquisas relacionadas ao uso de SIG no turismo vem aumentando. Conclui-se que, graças aos avanços tecnológicos com o uso de WebGIS e GIS de código aberto, os gerentes de negócios de turismo puderam construir sistemas de informações sobre recursos turísticos.

Palavras-chaves: inteligência comercial, sistemas de apoio à decisão, turismo, sistemas de informação geográfica e SIG.

\section{Introducción}

En el contexto de la realidad empresarial a nivel mundial, la utilización de sistemas de información es una estrategia corporativa que ha tenido un gran impacto, pues constituye un factor clave del éxito organizacional, permitiendo alcanzar ventajas competitivas significativas (Muñoz, Osorio y Zúñiga, 2016).

El surgimiento de tecnologías como los Sistemas de Información Geográfica (SIG) han servido "como un referente... para el desarrollo tecnológico en diferentes áreas como la agricultura, ganadería, hidrografía, turismo, topografía" (Ulloa, Orozco, Orozco y Carrera, 2017), puntualmente, en el sector turístico son de gran utilidad ya que permiten conocer los intereses de las personas que visitan diferentes zonas, 
acorde al enfoque territorial y cultural que tiene cada región (Ulloa et al., 2017). Por otra parte, la inteligencia de negocios es una herramienta usada para definir estrategias a implementar en las diversas áreas de las empresas en pro de su competitividad y sostenibilidad, mediante la extracción, el procesamiento y el análisis de la información (Rus, 2008), de manera que el conocimiento generado pueda tener aplicaciones prácticas y efectivas que se traduzcan en la toma de decisiones acertadas y que generen beneficios y posicionamiento.

Una de las áreas de aplicación de la inteligencia de negocios en el sector turístico es la industria hotelera, pues al usar los sistemas de información es posible proyectar datos a futuro y tener en cuenta los registros estadísticos de años anteriores en cuanto a cantidad de turistas, preferencias con respecto a los tipos de turismo, potencialidades de zonas turísticas, entre otros; de manera que se puedan analizar tendencias y maximizar ganancias (Cabiddu, Lui y Piccoli, 2013).

En líneas generales, las aplicaciones de SIG en turismo son diversas, por ejemplo, "para establecer el potencial turístico de un territorio, la primera fase es determinar los indicadores turísticos que lo hacen atractivo mediante los Métodos de Decisión Multicriterio" (González, Goncalves y Naranjo, 2016). A partir de ello, en una segunda fase, "se establecen los datos cuantitativos mediante los Sistemas de Información Geográfica (SIG), que son considerados como una herramienta de gran alcance en investigaciones y gestión de recursos" (González et al., 2016).

En este orden de ideas, (Bocalandro y Krauthamer, 2007) refieren que:

han surgido muchas aplicaciones GIS como por ejemplo guías turísticas construidas por aficionados a la informática, o tal vez porque no poder conocer el recorrido de una las pruebas ciclísticas más conocidas como lo es el tour de Francia y además poder hacer un seguimiento de la carrera en tiempo real.
Sin duda, resulta interesante que mediante la inteligencia de negocios se pueden analizar a los clientes potenciales, sus preferencias y demás datos que alimentan a la industria, los cuales pueden hacerla funcionar de una manera óptima, obteniendo un beneficio mutuo entre cliente y sector, tanto turístico como hotelero (Korte, Ariyachandra y Frolick, 2013).

Con base en lo expuesto, este artículo tiene por objetivo realizar una revisión bibliográfica sobre el uso de sistemas de información geográfica en turismo para la toma de decisiones apoyadas en la inteligencia de negocios. Lo que, a su vez, permitirá contar con un antecedente investigativo de carácter científico, que brinde una noción amplia de los avances realizados en esta área durante los últimos años, destacando los principales indicadores y factores que impulsan el uso de los SIG en el ámbito turístico.

\section{Herramientas, modelos y métodos para la gestión de la información en el entorno turístico}

El surgimiento de nuevas tecnologías ha transformado el manejo de la información en el sector turístico especialmente con la inclusión de la inteligencia de negocios y big data. Diversos artículos exponen enfoques de gran utilidad para mejorar la productividad y garantizar la competitividad del sector turístico. Sería apropiado mostrar las ventajas de cada técnica expuesta en los artículos, para así realizar una comparación sobre los diferentes métodos empleados y obtener conclusiones acertadas y pertinentes para el trabajo a desarrollar.

Se emplean técnicas como el aprendizaje no supervisado de modelado de temas que permite evaluar las opiniones de los clientes, análisis de texto que presenta gran utilidad en la generación de mapas dados por los términos más frecuentes, también plataformas de procesamiento de lenguaje natural que proporcionan tecnologías de procesamiento de texto como análisis de sentimiento, 
opinión pública y reconocimiento de entidad nombrada (NER).

Es necesario tener en cuenta que hay diversos enfoques y técnicas que permiten extraer información de los clientes, pero también es importante analizarla para identificar la que se adapta más a lo que la empresa turística desea brindar a sus clientes potenciales.

La inteligencia de negocios presenta una notable importancia en el sector hotelero, ya que permite un aumento de la efectividad. No obstante, se considera una limitación actual de las industrias que incorporan inteligencia de negocios en sus procesos empresariales, la mantenibilidad del estado competitivo alcanzado, una vez que ya han incorporado iniciativas estratégicas Tl; por lo que es fundamental evaluar la manera de extraer, procesar y analizar cada uno de los datos; se mencionan los cubos analíticos de procesamiento en línea (OLAP) que presentan una destacada ventaja en cuanto a la generación de informes. El análisis de datos permite comprender la cohesión en lugar de considerar problemas individuales, mediante el método Ilamado Failure Mode Effects Análisis (FMEA).

El futuro de la inteligencia de negocios en la industria hotelera puede ser prometedor, ya que brinda ayuda a los hoteles para mirar datos a futuro y tener en cuenta los del pasado, y así analizar tendencias y maximizar ganancias. Asimismo, la inteligencia de negocios permite tener en cuenta las preferencias y los datos de los clientes potenciales, lo cual propicia que la industria funcione de una manera óptima, obteniendo un beneficio mutuo entre clientes y sector hotelero.

Alzua-Sorzabal, Rebon y Kepa (2016) explican la importancia de usar tecnologías en el sector turístico que propicen aumentar el rendimiento y mejorar la percepción por medio de la información recolectada, por ejemplo, la herramienta web MWD (Monitor Web Destination) permite medir, analizar y modelar el comportamiento de los visitantes y promueve un destino turístico. Las herramientas de análisis web promueven técnicas como la minería web aplicadas al sector turístico y la técnica KDD (Knowledge Discovery in Databases), que permite descubrir información útil dentro de todos los datos obtenidos en un repositorio.

De tal modo que los sistemas inteligentes en el sector turismo como MWD ayudan a las organizaciones a visualizar una unión de tecnologías en los negocios que permite realizar acciones inteligentes para abordar procesos convencionales. El uso de sistemas de apoyo, basados en inteligencia de negocios, coadyuvan a mejorar notablemente la productividad y la eficiencia, ya que a través de ellos se pueden tener en cuenta diferentes tipos de indicadores que respalden los procesos empresariales.

La clasificación y la medición de la información se puede expresar por palabras o calificaciones; múltiples industrias, con el apoyo de técnicas de inteligencia de negocios, utilizan BI en sus procesos de recomendación e indexación de contenidos o productos, esto les sirve a sus clientes como sugerencia o acercamiento, el caso del turismo no es la excepción.

Desde la perspectiva de Alzua-Sorzabal (2016), la indexación y la clasificación de recomendaciones no están unificadas y, en ocasiones, no se tiene un criterio claro en cuanto a calificación o evaluación con base en los productos o servicios ofertados en materia turística, por lo que se debe tener en cuenta que las recomendaciones están presentes en cualquier contexto y estas se deben interpretar y diferenciar, dependiendo del criterio de cada usuario, pero aun así se necesitan tener herramientas y técnicas acordes a las nuevas tecnologías y a los nuevos retos que presenta el turismo. Se presentan avances conceptuales que se integran en el diseño de un sistema de indicadores para la medición de IM (Innovation Management).

Por medio de esta estrategia se busca generar una rápida difusión y uso del conocimiento tecnológico, para adaptarse a diversos factores que afectan los procesos y como resultado de esto, optimizarlos para aumentar la competitividad. A pesar de todas las investigaciones que se han realizado, no se describe un modelo que se haya 
aceptado. Como métodos necesarios se implementan la inteligencia de negocios y el BSC (Balanced Scorecard), los cuales proporcionan la estructura necesaria para un sistema de gestión y medidas de desempeño para medición estratégica.

Por medio de este estudio se confirma la importancia de medir IM en las organizaciones turísticas y tener herramientas apropiadas para dicha actividad. Es necesario tener en cuenta los cambios que influyen en el desempeño, para este caso específico del sector turístico, y así identificar herramientas que permitan evaluar estos cambios y evitar impactos tan significativos en el rendimiento organizacional (López, 2009).

\section{Inteligencia de negocios en el turismo}

El uso de inteligencia de negocios (IN) ha contribuido a la mejora de la competitividad en la industria del turismo a lo largo del tiempo. Esta se ha beneficiado de las ventajas que conlleva la adopción de un sistema de IN, tales como las capacidades de captura, almacenamiento, recuperación, procesamiento y análisis de datos de turistas flexibles y fáciles de usar, entre otras (Nyanga, Pansiri y Chatibura, 2019).

Gracias a los avances tecnológicos, la inteligencia de negocios (IN) en el turismo ha tenido un gran desarrollo durante los últimos años. En este contexto, se destaca el uso del contenido generado por el usuario para la IN, específicamente, los sitios web de revisión por pares están ganando importancia en la toma de decisiones de los viajeros y proporcionan información para la gestión de los destinos. Las revisiones textuales son especialmente importantes, pero muy extensas y difíciles de procesar. Es aquí donde la aplicación de la metodología de lingüística computacional a la literatura turística ha brindado grandes beneficios, proporcionando un análisis exhaustivo de los datos que pueda ser utilizado para cumplir metas empresariales (Godnov y Redek, 2019).

Otra aplicación que ha tenido relevancia ha sido la determinación automática basada en la inteligencia artificial híbrida de las preferencias de viaje de los turistas. Se observó que es posible encontrar a los turistas que prefirieren un país frente a otro utilizando este tipo de sistema. El mismo evidencia que, los países pueden llegar al individuo en lugar de a las masas en sus actividades de promoción (Topal y Uçar, 2019).

En el contexto colombiano, la IN aplicada al ecoturismo destaca por la utilización de un "Sistema de Información para el Turismo de Naturaleza en Colombia, donde mediante la utilización de la metodología CRISP-DM, se llega a un prototipo funcional que permite la visualización de diferentes variables relevantes para el ecoturismo, con el fin de identificar oportunidades y brechas de los departamentos de Colombia" (Betancur, Gómez y Quintero, 2019).

\section{Metodología}

La investigación se desarrolló bajo la modalidad de revisión sistemática de literatura, la cual:

es un artículo de "síntesis de la evidencia disponible», en el que se realiza una revisión de aspectos cuantitativos y cualitativos de estudios primarios, con el objetivo de resumir la información existente respecto de un tema en particular. Los investigadores luego de recolectar los artículos de interés; los analizan, y comparan la evidencia que aportan con la de otros similares" (Manterola y Astudillo, 2013, p. 149).

Dicha revisión, se propuso estudiar el uso de los SIG en turismo en el marco de la inteligencia de negocios. El proceso investigativo se dividió en tres etapas:

\section{Selección de la fuente de información y selección de los datos de la muestra}

Estuvo representada por XX artículos de revistas científicas indexados en Scielo, Redalyc.org, ELSEVIER, ScienceDirect, entre otras. Se utilizó la base de datos Scopus e IEEE. 
Las ecuaciones de búsqueda (se utilizaron en el campo Topic) fueron:

(TITLE-ABS-KEY (tourism AND Business Intelligence)

(TITLE-ABS-KEY (tourism AND geographic information systems)

(TITLE-ABS-KEY (tourism AND SIG AND Business Intelligence)

Como criterios de inclusión se seleccionaron artículos realizados desde 2015 hasta 2019, redactados en idioma inglés y español que abordan el tema de alguna de las variables bajo estudio: Sistemas de información geográfica, inteligencia de negocios o turismo.

\section{Transformación de los datos mediante el uso de técnicas bibliométricas}

Se utilizó el programa VOSviewer a través del cual se generaron mapas creados que incluyen elementos que hacen referencia a los tópicos de interés, tales como: tipo de publicaciones, investigadores, términos, país de realización, autores, entre otros. Esta herramienta permite exportar los datos de Web of Science, Scopus, PubMed, RIS, Crossref, entre otras (Eck y Waltman, 2019).

La herramienta provee tres formas de visualización: de la red, de superposición y la de densidad. En la primera, cada elemento está representado por una etiqueta y un círculo. Los vínculos de co-citación más fuertes están representados por líneas, en general, cuanto más cerca se encuentran dos ítems, más fuerte es su relación. El color del ítem depende del clúster en el cual se halla clasificado.

Por otro lado, la representación en red de superposición es similar al caso anterior, pero en ella el color del ítem cambia teniendo en cuenta el año en el cual hubo mayor actividad científica relacionada con el objeto de estudio. Por último, la visualización de densidad de cada ítem posee un color que indica la densidad de los elementos en ese punto; cuanto mayor sea el número de otros elementos alrededor de un ítem y mayor sea el peso de estos, el color asociado tenderá a ser amarillo.

\section{Reporte de los resultados}

Para la representación se resultados se emplearon gráficos de creación propia en conjunto con los mapas de clusters y densidad de relaciones entre palabras clave generados por VOS viewer.

\section{Resultados y discusión}

De acuerdo con la bibliografía evaluada, son numerosas las investigaciones que han tratado el tema del uso de los GIS en turismo, en este sentido, seguidamente se presentan los principales hallazgos encontrados.

\section{Turismo y GIS}

Al realizar el análisis de la información obtenida, luego de la aplicación de la ecuación de búsqueda en las bases de datos Scopus e IEEE, se procedió a tratar esta información en el programa VOSviewer, en donde se obtuvo un mapa de relación de la cantidad de publicaciones realizadas por año en función de las variables GIS y Turismos (Figura 1).

Como puede observarse, durante el periodo 2015 a 2019 la tendencia de publicación es ligeramente creciente en relación con la temática del uso de SIG en turismo. Al mismo tiempo, en la Figura 2 se ilustran las principales relaciones de palabras clave establecidas:

De la figura anterior se deriva que la tecnología GIS (gis technology) se encuentra relacionada con estrategias de e-turismo (e-tourism) empleadas en la gestión de la información (information management) de la industria de viajes (travel industry), la cual, los utiliza para el estudio de data bases espaciales (spatial databases) que permitan realizar una planificación (planning) eficiente en el marco rural y urbano (town and country planning), relacionado con el turismo. Igualmente, son utilizados 


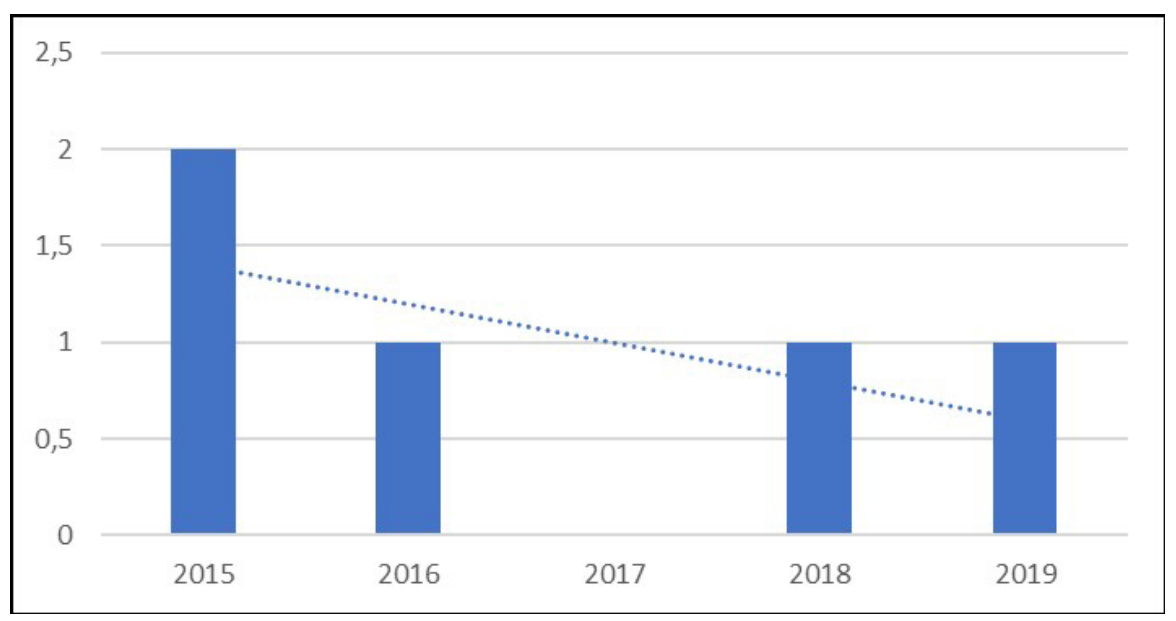

Figura 1. Tendencia anual de publicación de artículos relacionados con SIG y turismo.

Fuente: Los autores.

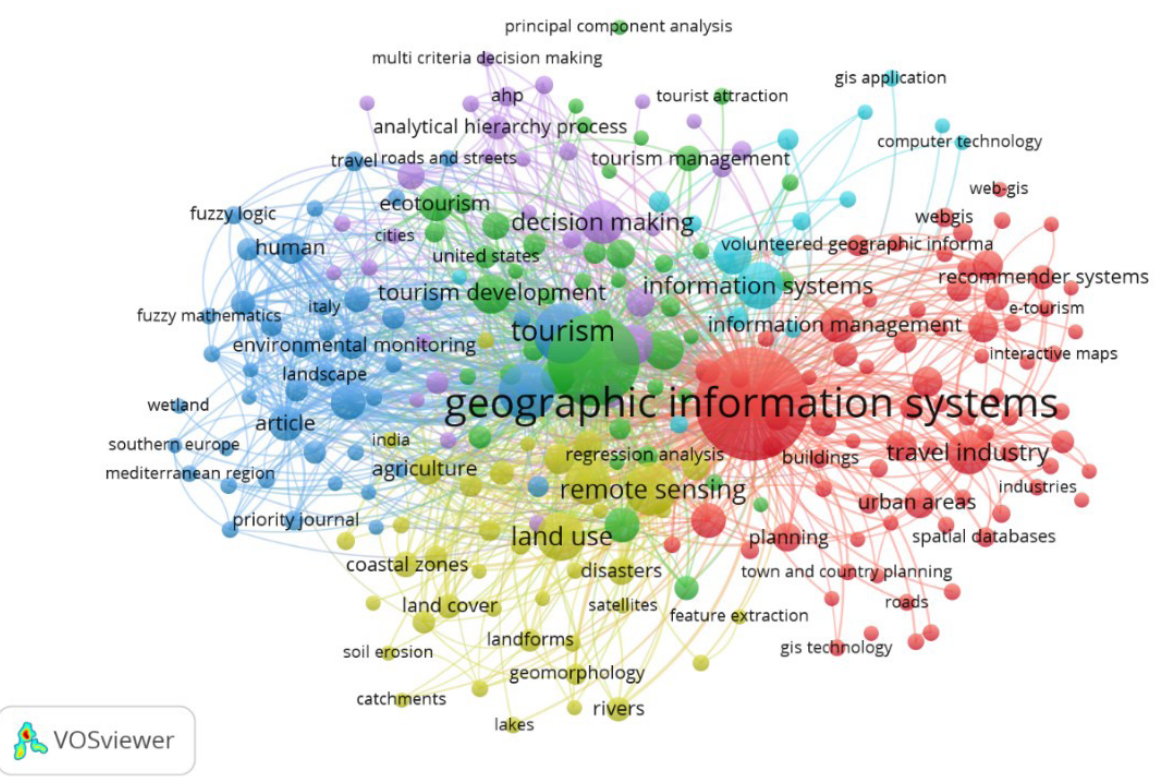

Figura 2. Mapa de clusters de las relaciones estrechas entre palabras clave resultantes de la fórmula de búsqueda:

(TITLE-ABS-KEY (turism AND geographic information systems).

Fuente: Los autores a partir de VOSviewer.

como sistemas de recomendación (recommender systems) de destinos turísticos.

Al mismo tiempo, se tiene que este tipo de sistemas permite realizar monitoreo ambiental (environmental monitoring) de paisajes (landscape) que se encuentren en sitios turísticos de gran potencial, que puedan ser fuente de crecimiento económico y desarrollo turístico (tourism development) para las regiones donde se encuentren ubicados.
Gracias al uso de la tecnología computacional (computer technology) ha sido posible el desarroIlo de aplicaciones GIS (gis application). La utilidad de los GIS en el ámbito turístico ha impulsado el desarrollo de una gran cantidad de investigaciones relacionadas con esta temática, en función de esto, en el siguiente gráfico se ilustra el mapa de densidad relacional entre las palabras claves utilizadas en los artículos científicos consultados. 


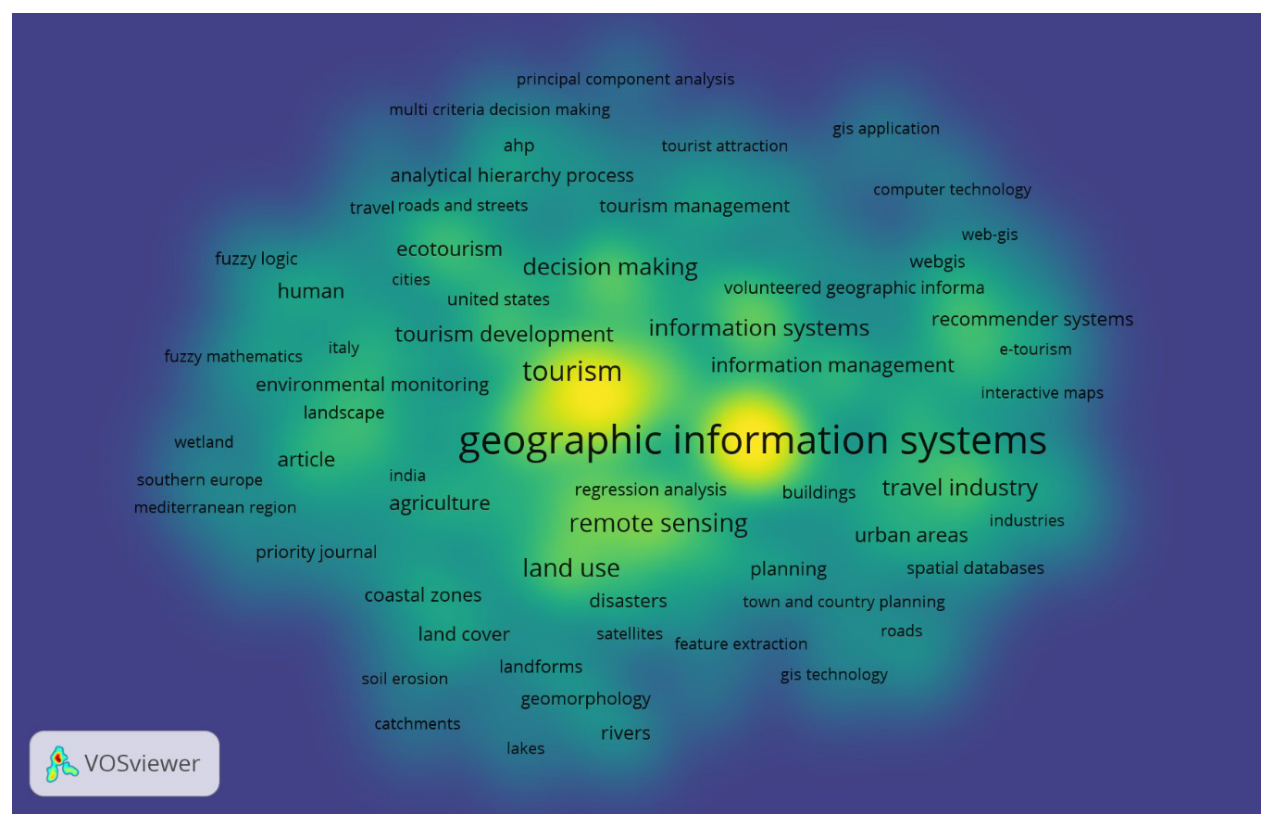

Figura 3. Mapa de densidad de las relaciones estrechas entre palabras clave resultantes de la fórmula de búsqueda: (TITLE-ABS-KEY (turism AND geographic information systems).

Fuente: Los autores a partir de VOSviewer.

La variable turismo (tourism) se encuentra estrechamente relacionada con los GIS (geografic infomation systems), puesto que la densidad relacional entre los artículos que abordan dichas palabras claves es alta. Revisando la literatura asociada a dichas variables, se obtuvo una de las aplicaciones de los SIG en turismo en el marco de la inteligencia de negocios es la implementación de un sitio de turismo de negocios utilizando un SIG, esta web apoya a tres sectores: gubernamental, sociedad y de tours (Taha, Rashid y Sallam, 2017).

En un estudio donde se analizó el uso de los SIG para determinar el potencial del turismo rural, se optó por utilizar el "QGis1, por ser un (...) SIG) de código abierto que permite trabajar en varios sistemas operativos, formatos diferentes, con multitud de bibliotecas, además de permitir integrar aplicaciones en lenguaje Python, lo que posibilita obtener aplicaciones específicas" (González et al., 2016).

Asimismo, se tiene que el rápido desarrollo del turismo ha creado grandes desafíos en la planificación y la gestión del medio ambiente. Esto se debe a la necesidad de proporcionar sitios turísticos atractivos y productos de apoyo como alojamientos y redes de transporte, así como proteger el medio ambiente. En este contexto, se han utilizado los SIG para mapear y medir la transformación del uso de la tierra y examinar áreas que probablemente experimentarán presión de desarrollo urbano. Las estrategias de zonificación como contener áreas urbanas dentro de las áreas urbanas existentes y promover el desarrollo sostenible del turismo en diversas regiones han logrado controlar la expansión de las áreas urbanas (Hasshim Rahman, Khalid, Manan, 2015).

Aunado a esto, el sector turístico ha contribuido significativamente a la economía y al desarrollo socioeconómico en diversas regiones. Además, los sitios potenciales para el desarrollo urbano identificados, utilizando el enfoque MCE basado en SIG, también han revelado que se pueden desarrollar miles de acres para diversas actividades, incluido el turismo. Dicho enfoque, proporciona un marco y una guía para los planificadores y tomadores de decisiones en la planificación y el desarrollo de tierras para actividades turísticas en las regiones que se han implementado (Hasshim et al., 2015). 


\section{Inteligencia de negocios y turismo}

Al analizar las variables inteligencia de negocios y turismo se observa que las interrelaciones son mucho menores en comparación con la búsqueda anterior; en la Figura 4 se ilustran los resultados obtenidos.

Aunado a lo anterior, en la Figura 5 se representa el mapa de densidad de las relaciones estrechas entre palabras clave resultantes de la fórmula de búsqueda (TITLE-ABS-KEY (tourism AND Business Intelligence).

En este marco, la inteligencia artificial (artificial intelligence) constituye el principal indicador abordado en las investigaciones consultadas. Destacando que el pronóstico de la llegada de visitantes es de gran importancia ya que es un indicador de la demanda turística y puede servir como referencia para las políticas gubernamentales sobre el turismo y las estrategias comerciales de las industrias turísticas. En este contexto, la inteligencia de negocios ha sido útil para la creación de un modelo de pronóstico preciso (Chang y Tsai, 2017).

Igualmente, en cuanto a los sistemas de soporte de decisiones (decision support system), destaca que, dado el crecimiento en el nivel de competitividad en las empresas de turismo, la mayoría de las organizaciones buscan estrategias efectivas para aumentar la escala de la base de clientes de los mercados locales e internacionales enfocándose en la satisfacción del cliente, ya que es un indicador clave de la intención de recompra y la lealtad del cliente. Debido a esta razón, las empresas siempre brindan a los clientes canales de comunicación como el correo electrónico para percibir las experiencias de los clientes. En consecuencia, se ha enviado una gran cantidad de correos electrónicos a las empresas (Chumwatana y Chuaychoo, 2016).

Sin embargo, extraer quejas de una gran cantidad de correos electrónicos en diferentes idiomas es muy difícil, por lo que surgió una metodología novedosa para extraer automáticamente los

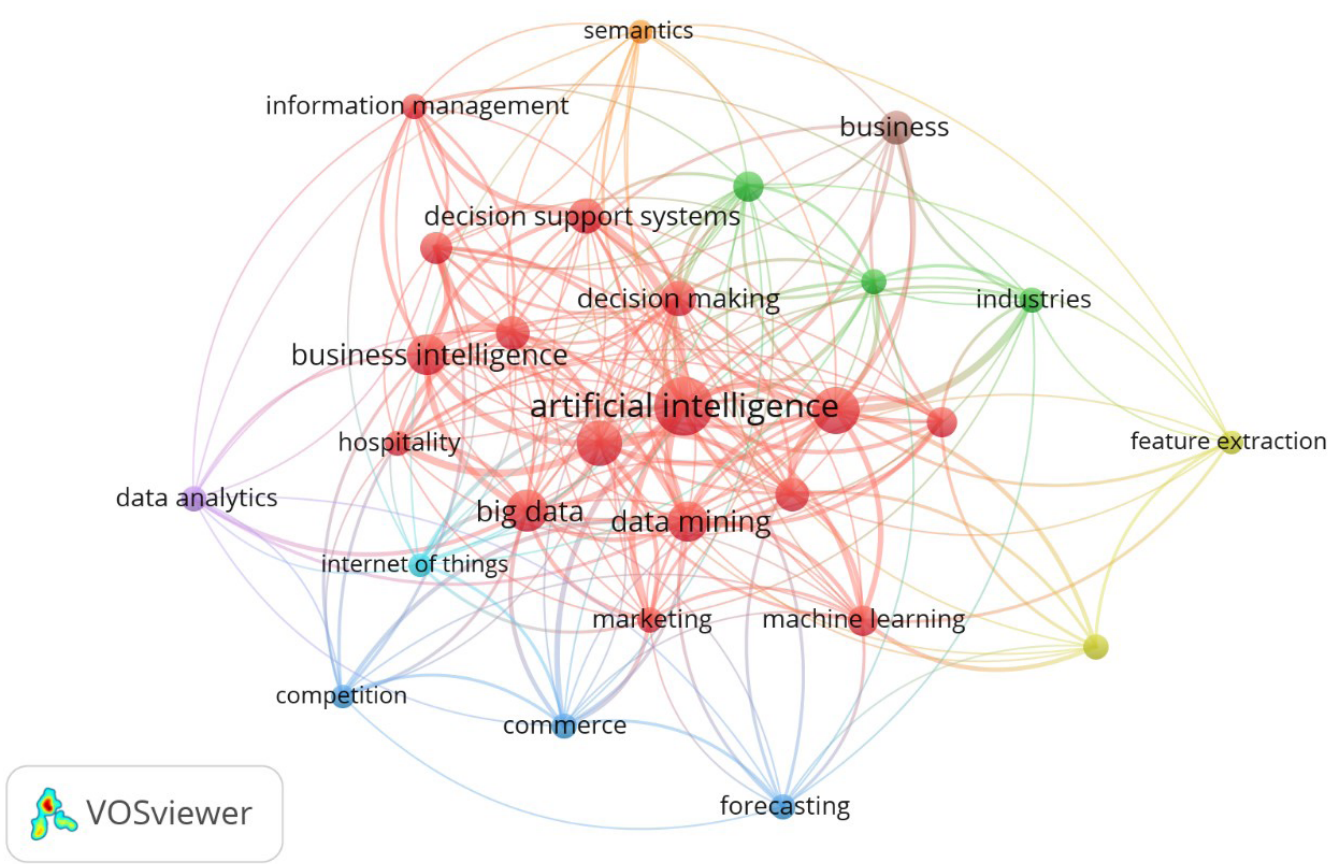

Figura 4. Mapa de clusters de las relaciones estrechas entre palabras clave resultantes de la fórmula de búsqueda: (TITLE-ABS-KEY (turism AND Business Intelligence).

Fuente: Los autores a partir de VOSviewer. 


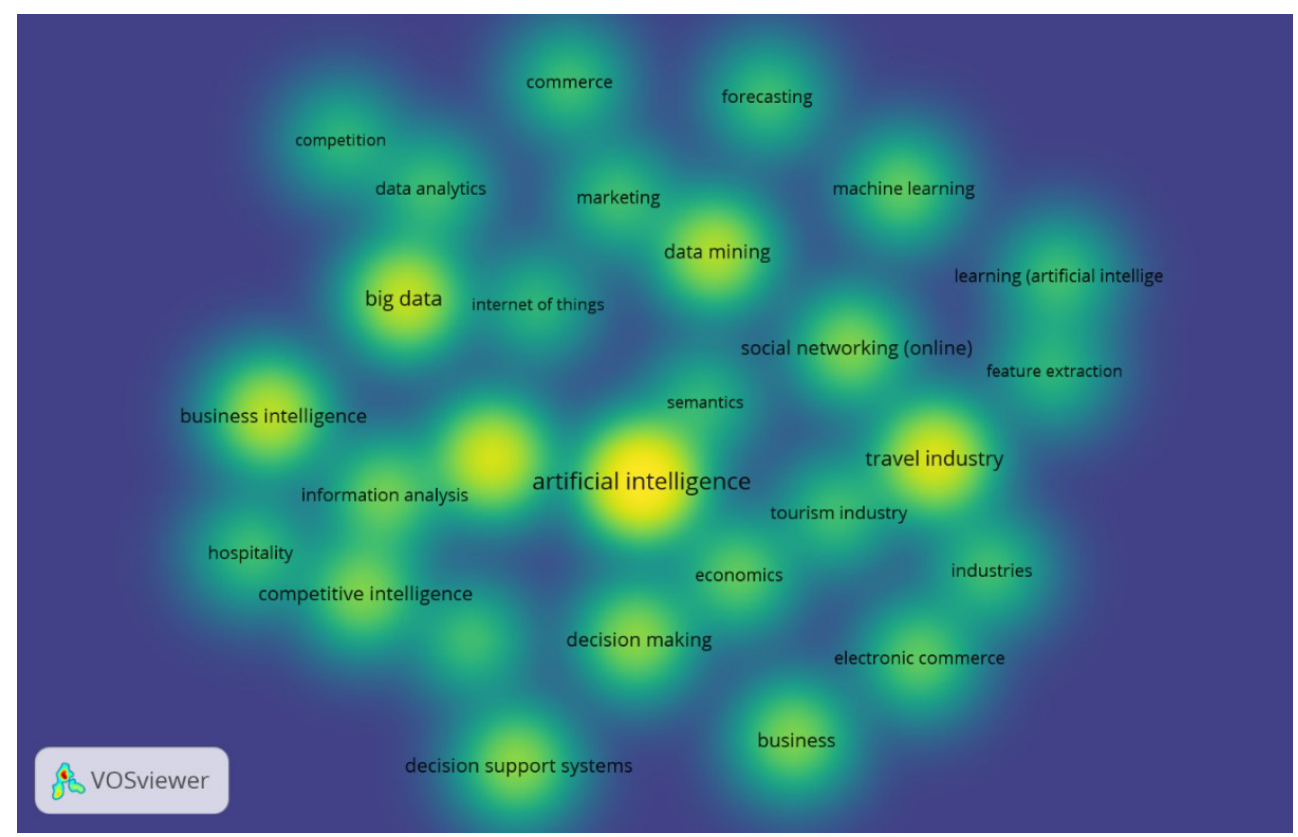

Figura 5. Mapa de densidad de las relaciones estrechas entre palabras clave resultantes de la fórmula de búsqueda (TITLE-ABS-KEY (tourism AND Business Intelligence).

Fuente: Los autores a partir de VOSviewer.

correos electrónicos de quejas de la bandeja de entrada para cualquier idioma. La extracción de $\mathrm{N}$-gram, que es independiente del lenguaje, y la técnica de clasificación se han aplicado en la técnica propuesta. Los resultados experimentales muestran que el rendimiento de clasificación de la técnica propuesta es aproximadamente del 90 $\%$. Esto muestra ventajas significativas para que las empresas de turismo respondan de inmediato a los clientes que tienen experiencias negativas, a fin de mantener una buena relación y obtener un mayor número de clientes (Chumwatana y Chuaychoo, 2016).

\section{Turismo, inteligencia de negocios y GIS}

Con respecto a las investigaciones realizadas abordando las tres variables bajo estudio (turismo, SIG e inteligencia de negocios), se observa que la producción científica ha sido muy poca, en la Figura 6 se ilustran la cantidad de artículos publicados por año durante el periodo 2016-2019.
Como se observa, la producción científica que integra la investigación en función de SIG, turismo e inteligencia de negocios ha sido limitada durante los últimos cinco años, se muestra una tendencia decreciente desde el 2015 hasta el 2019. Al integrar a la búsqueda la variable Business Intelligence se establecen nuevas relaciones, en la Figura 7 se ilustran los clusters de las relaciones estrechas entre palabras clave resultantes de la fórmula de búsqueda: (TITLE-ABS-KEY (turism AND SIG AND Business Intelligence).

Uno de los hallazgos más significativos de las investigaciones consultadas es que, en el marco de la inteligencia de negocios, se puede utilizar un sistema de gestión de recursos turísticos de alguna región basado en SIG para proporcionar apoyo técnico para la gestión turística; mediante la base de datos de Access, que utiliza la tecnología SIG en la plataforma de desarrollo VB para el desarrollo del sistema, este tiene las ventajas de un ciclo de desarrollo corto, simple y rápido. El sistema puede proporcionar servicios de gestión de 


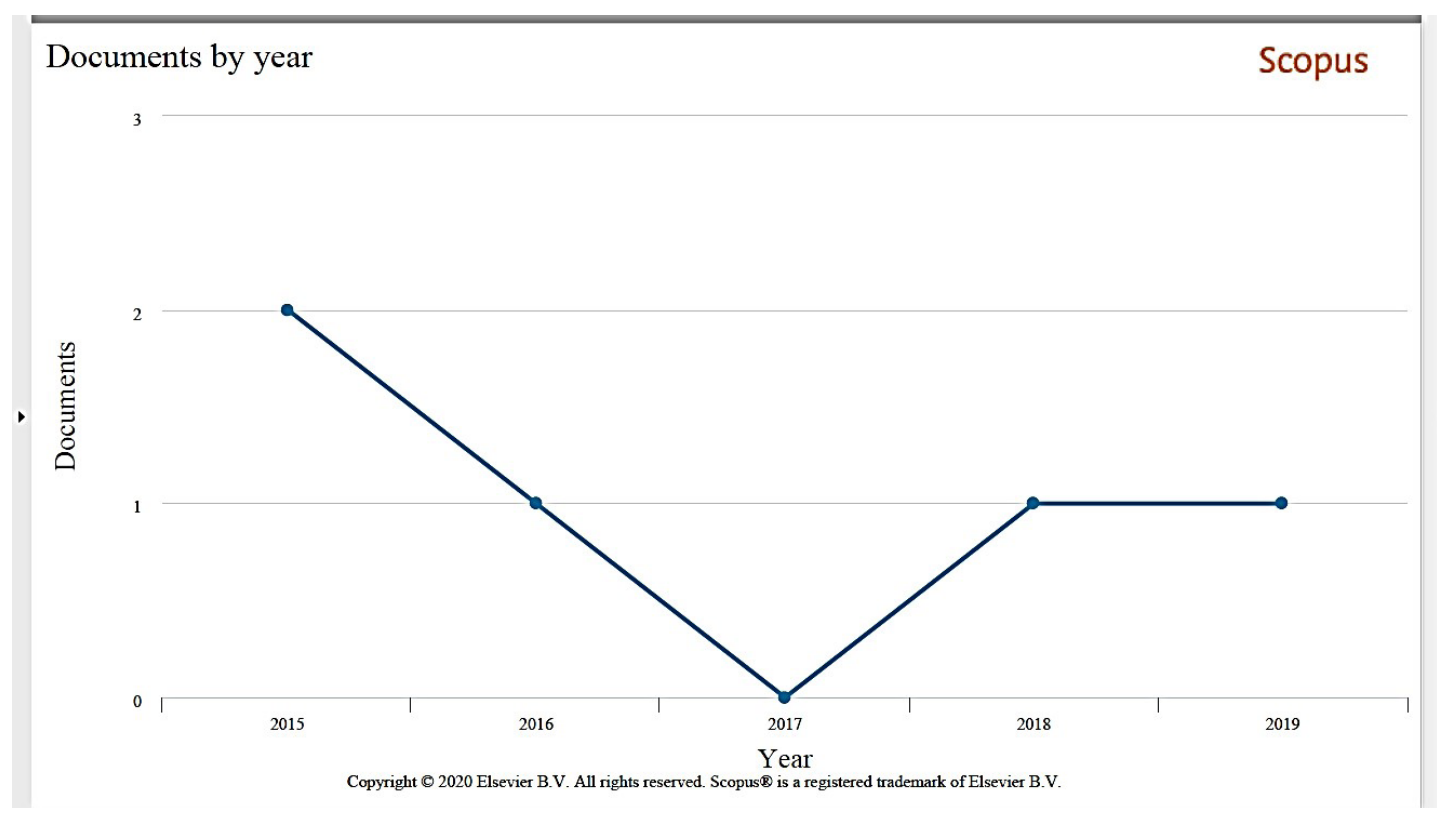

Figura 6. Tendencia anual de publicación de artículos relacionados con Inteligencia de negocios, SIG y turismo. Datos obtenidos de Scopus.

Fuente: Scopus.

A vosviewer

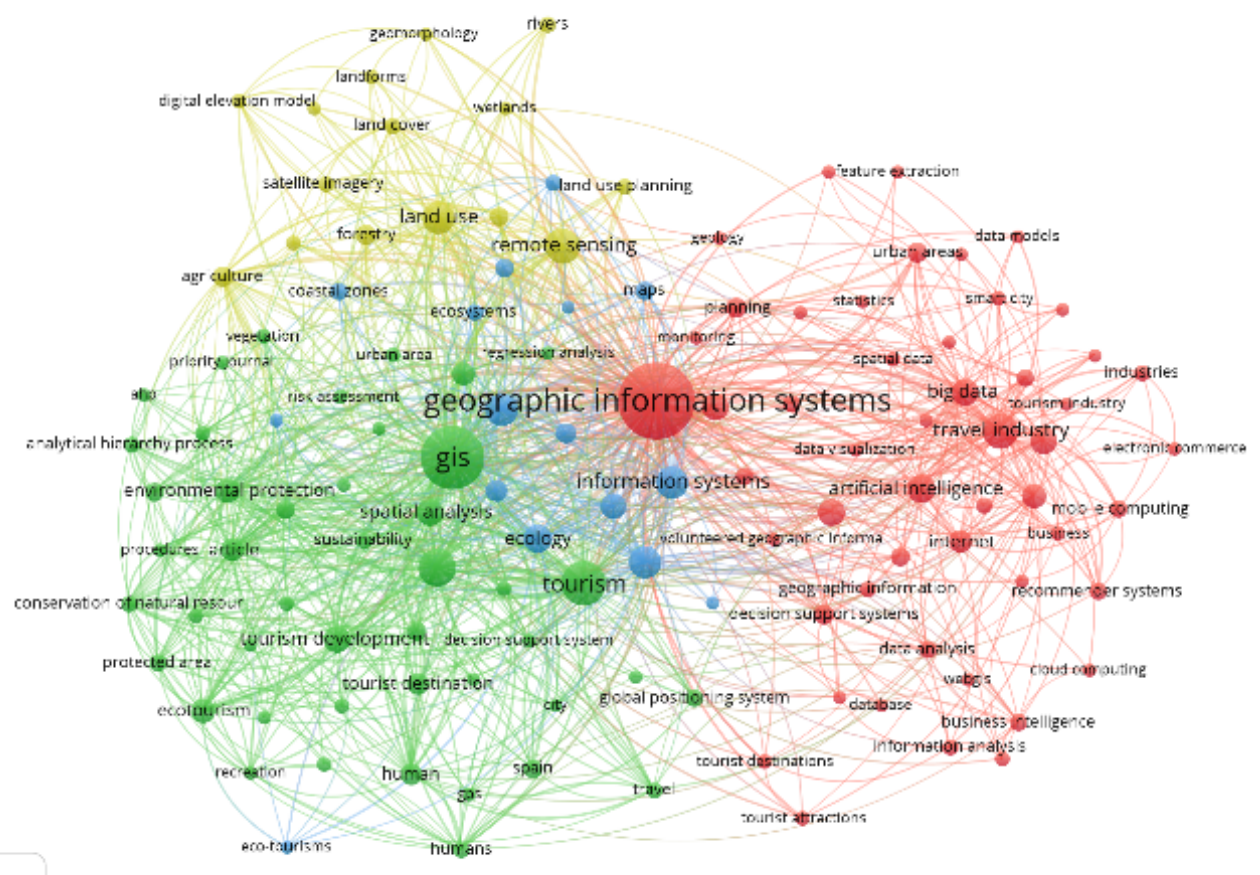

Figura 7. Mapa de clusters de las relaciones estrechas entre palabras clave resultantes de la fórmula de búsqueda: (TITLE-ABS-KEY (turism AND SIG AND Business Intelligence).

Fuente: Los autores a partir de VOSviewer. 
consultas de información detallada para satisfacer las necesidades de los gerentes (Ling, 2017).

Con el rápido desarrollo de redes y tecnología informática, WebGIS ha cambiado el modo tradicional de aplicación SIG, haciéndose cada vez más popular, incluso en el ámbito de las industrias turísticas. Al mismo tiempo, los proyectos de software Open Source GIS y los estándares técnicos abiertos se han desarrollado rápidamente. Durante el proceso de informatización en diferentes campos, la mayoría de los gerentes utilizan una plataforma de software SIG comercial o algún software SIG de código abierto que ha tenido una larga historia y un marco sofisticado para construir su sistema de gestión y servicio de información. Con base en el análisis de la tecnología Open Source GIS, se presentó un nuevo esquema de diseño WebGIS basado en la tecnología de middleware GIS Open Source. Y luego se usó un Sistema de Información de Recursos Turísticos como ejemplo para verificar la viabilidad de este proyecto (Lv et. al., 2016).

También, se ha hecho uso del sistema de información geográfica y análisis de criterios múltiples para el diseño de rutas de viaje (Arthan et al., 2019). En este marco, se tiene que una de las formas más efectivas para mejorar la calidad de la gestión de las reservas naturales es integrar en profundidad todos los recursos de información basados en la tecnología de información geográfica espacial moderna y construir una plataforma de gestión de información digital de reservas naturales (Wu, Wangh, Zhong, Da y Zha, 2015).

\section{Conclusiones}

En los últimos años, la tendencia en investigaciones referentes a GIS y turismo ha sido creciente, utilizada en estudios que abordaron la temática de implementación de sitios de turismo de negocios utilizando un SIG, enmarcados en sectores de tipo gubernamental, social y de turismo (Taha, Rashid y Sallam, 2017). Igualmente, se ha abordado el tema del uso de GIS para estimar el potencial del turismo rural (González, Goncalves y Naranjo, 2016).
Todo ello, motivado por el hecho de que el sector turístico ha contribuido de forma significativa a la economía y desarrollo socioeconómico en diversas regiones a lo largo del mundo. Destacando que los sitios potenciales para el desarrollo urbano identificados utilizando el enfoque MCE basado en SIG han revelado que es posible desarrollar amplias extensiones de territorio para actividades turísticas (Hasshim et. al., 2015).

Sin embargo, al indagar sobre la producción científica de los últimos 5 años, que comprende el periodo 2015 a 2019, los estudios y artículos investigativos que incorporen la inteligencia de negocios en el marco de los SIG y su uso en turismo ha sido escasa. Los aportes realizados han sido en materia de sistemas de gestión de recursos turísticos de diversas regiones basados en SIG (Ling, 2017), Sistema de Información de Recursos Turísticos (Lv et al., 2016) y plataformas de gestión de información digital de reservas naturales (Wu et al., 2015).

\section{Referencias}

Alzua-Sorzabal, A., Rebon F., Kepa, J. (2016), El Observatorio Turístico Avanzado, un sistema de Inteligencia Competitiva basado en Internet. San Sebastián: Centro de Investigación Cooperativa en Turismo.

Arthan, S., Pimonsree, L., Ket-ord, R., Ruelom, A., Thongpet, S., Kantawong, K. (2019). Using Geographical Information System and Multi-Criteria Analysis for Travelling Routing Design from Phayao Province to Luang Prabang. The 4th International Conference on Digital Arts, Media and Technology and 2nd ECTI Northern Section Conference on Electrical, Electronics, Computer and Telecommunications Engineering, Nan, Thailand. https://doi. org/10.1109/ECTI-NCON.2019.8692294

Ballesteros-Ballesteros, V. A. (2019). La educación en energías renovables como alternativa de promoción del compromiso público ascendente entre los Indígenas Wayuu en la Alta 
Guajira. Revista Científica, 388-397. https:// revistas.udistrital.edu.co/index.php/revcie/ article/view/14773

Betancur, S., Gómez, M., Quintero, J. (2019). Inteligencia de negocios aplicada al ecoturismo en Colombia. Un caso de estudio aplicando la metodología CRISP-DM. 14th Iberian Conference on Information Systems and Technologies (CISTI), Coimbra, Portugal.

Bocalandro, N., Krauthamer, D. (2007). Geomarketing: aplicaciones de sistemas de información geográficos e inteligencia de negocios. $2^{\circ}$ Congreso Metropolitano de Ciencias Económicas, Ciudad Autónoma de Buenos Aires.

Cabiddu, F., Lui, T., Piccoli, G. (2013). Managing Value Co-Creation in the Tourism Industry, $A n$ nals of Tourism Research, 42(1), 86-107. https://doi.org/10.1016/j.annals.2013.01.001

Callejas-Cuervo, M., Martínez-Tejada, L., \& Alarcón-Aldana, A. (2017). Emotion recognition techniques using physiological signals and video games -Systematic review-. Revista Facultad de Ingeniería, 26(46), 19-28. https://doi. org/10.19053/01211129.v26.n46.2017.7310

Camargo, J., Joyanes, L., Giraldo, L. (2016). La inteligencia de negocios como una herramienta en la gestión académica. Revista Científica, 24, 110-120. https://doi.org/10.14483/10.14483/ udistrital.jour.RC.2016.24.a11

Chang, Y., Tsai, C. (2017). Apply deep learning neural network to forecast number of tourists. 31 st International Conference on Advanced Information Networking and Applications Workshops, Taipei. https://doi.org/10.1109/ WAINA.2017.125

Chumwatana, T., Chuaychoo, I. (2016). Automatic Filtering Non-English Complaint Emails in Tourism Industry Using Ngram Extraction and Classification Techniques. 4th International Symposium on Computational and Business Intelligence, Olten. https://doi.org/10.1109/ ISCBI.2016.7743287

Eck, N., Waltman, L. (2019). Accuracy of citation data in Web of Science and Scopus. Paper published in the Proceedings of the 16th International Conference of the International Society for Scientometrics and Informetrics. Nueva York: Cornell University, 1087-1092.

Godnov, U., Redek, T. (2019) The use of user-generated content for business intelligence in tourism: insights from an analysis of Croatian hotels, Economic Research-Ekonomska Istraživanja, 32 (1), 2455-2480. https://doi.org/10.10 80/1331677X.2019.1633372

González, A., Goncalves, G., Naranjo, J. (2016). Uso de los SIG para determinar el potencial del Turismo Rural. Congreso Internacional de Turismo Rural y Desarrollo Sostenible, Santiago de Compostela.

Gutiérrez-Sabogal, L. H. (2016). Problemática de la educación ambiental en las instituciones educativas. Revista Científica, 3(23), 57-76. https:// doi.org/10.14483/udistrital.jour.RC.2015.23.a5

Hasshim, S., Rahman, A., Khalid, M. y Samad, A. (2015). Urban Development Pressure Towards Challenges in Ensuring Sustainable Tourism Development. IEEE International Conference on Control System, Computing and Engineering, Penang, Malaysia. https://doi. org/10.1109/ICCSCE.2015.7482250

Korte, D., Ariyachandra, T., Frolick, M. (2013). Business Intelligence in the Hospitality Industry. International Journal of Innovation, Management and Technology, 4(4), 429-434. https:// doi.org/10.7763/IIIMT.2013.V4.435

Ling, J. (2017). Zaozhuang Tourism Resource Management System Research Based on GIS. 3rd International Conference on Information Management. Zaozhuang, China.

López, A. (2009). Sistema integrado de gestión para empresas turísticas. Estudios Gerenciales, 25(112), 127-150. https://doi.org/10.1016/ S0123-5923(09)70083-X

Lv, D, Ying, X., Gao, X., Tao, W., Cui, Y., Hua, T. (2016). WebGIS Platform Design and Implementation Based On Open Source GIS Middleware. The $24^{\text {th }}$ International Conference on Geoinformatics, Galway, Ireland. 
Manterola, C. Astudillo, P., Arias, E., Claros, N. (2013). Revisiones sistemáticas de la literatura. Qué se debe saber acerca de ellas. Cirugia española. 91(3), 149-155. https://doi. org/10.1016/j.ciresp.2011.07.009

Muñoz, H., Osorio, M., Zúñiga, P. (2016). Inteligencia de los negocios. Clave del éxito en la era de la información. Clío América, 10(20), 194211. https://doi.org/10.21676/23897848.1877

Nyanga, C., Pansiri, J., Chatibura, D. (2019). Enhancing competitiveness in thetourism industry through the use of business intelligence: a literature review. Published in Journal of Tourism Futures. Emerald Publishing Limited. https://doi.org/10.21676/23897848.1877

Rojas-Montes, M., Pino-Correa, F., Martínez, J. (2015). Proceso de pruebas para pequeñas organizaciones desarrolladoras de software. Revista Facultad de Ingeniería, 24(39), 55-70. https://doi.org/10.19053/01211129.3551

Rus, G. (2008). Análisis Coste-Beneficio: Evaluación económica de políticas y proyectos de inversión. Ginés de Rus Ariel Economía, 3.a Edición, Barcelona, 2008, 372 págs.

Saavedra-Bautista, C., Cuervo-Gómez, W., Mejía-Ortega, I. (2017). Producción de contenidos transmedia, una estrategia innovadora.
Revista Científica, (28), 6-16. https://dx.doi. org/10.14483/udistrital.jour.rc.2016.28.a1

Taha, T., Rashid, S., Sallam, O. (2017). Web-based GIS Business Hotels Tourism Sites In Khartoum, Sudan. International Conference on Communication, Control, Computing and Electronics Engineering (ICCCCEE), Khartoum, Sudan.

Topal, I., Uçar, M. (2019). Hybrid Artificial Intelligence-Based Automatic Determination of Travel Preferences of Chinese Tourists. IEEE Access, 7, 162530-162548. https://doi. org/10.1109/ACCESS.2019.2947712

Ulloa, L., Orozco, F., Orozco, J., Carrera, F. (2017). Sistema de información geográfica para la integración de información Geo referenciada de entidades públicas y privadas en la ciudad de Santo Domingo. Revista Científica y Tecnológica UPSE, 4(3), 13-27. doi: https://doi. org/10.26423/rctu.v4i3.294

Wu, F., Wangh, J., Zhong, X., Da, M., Zha, Z. (2015). Web GIS-based Information Management System of Dashanbao Black-Necked Crane National Natural Reserve in Zhaotong, Yunnan. 2015 23rd International Conference on Geoinformatics, Wuhan, China. https://doi.org/10.1109/ GEOINFORMATICS.2015.7378610 